\title{
Using Doctoral Experience Survey Data to Support Developments in Postgraduate Supervision and Support
}

\author{
Lucy Johnston \\ University of Newcastle, \\ Callaghan, Australia
}

lucy.johnston@newcastle.edu.au

\author{
Keith Comer \\ Massey University, Palmerston \\ North, New Zealand
}

keith.comer@vuw.ac.nz

\author{
Kaylene A. Sampson \\ University of Canterbury, \\ Christchurch, New Zealand
}

kaylene.sampson@canterbury.ac.nz

\author{
Erik Brogt \\ University of Canterbury, \\ Christchurch, New Zealand \\ erik.brogt@canterbury.ac.nz
}

\begin{abstract}
Provision of both high standards of thesis supervision and high quality research environments are required for doctoral candidates to flourish. An important component of ensuring quality provision of research resources is the soliciting of feedback from research students and the provision from research supervisors and institutions of timely and constructive responses to such feedback. In this manuscript we describe the use of locally developed survey instruments to elicit student feedback. We then demonstrate how actions taken in response to this student feedback can help establish a virtuous circle that enhances doctoral students' research experiences. We provide examples of changes to supervisory practice and resource allocation based on feedback and show the positive impact on subsequent student evaluations. While the examples included here are local, the issues considered and the methods and interventions developed are applicable to all institutions offering research degrees.
\end{abstract}

Keywords: supervision; surveys; feedback; resource allocation; student evaluation

\section{Introduction}

Doctoral candidates contribute significantly to the academic life and reputation of universities.

Material published as part of this publication, either on-line or in print, is copyrighted by the Informing Science Institute. Permission to make digital or paper copy of part or all of these works for personal or classroom use is granted without fee provided that the copies are not made or distributed for profit or commercial advantage AND that copies 1) bear this notice in full and 2) give the full citation on the first page. It is permissible to abstract these works so long as credit is given. To copy in all other cases or to republish or to post on a server or to redistribute to lists requires specific permission and payment of a fee. Contact Publisher@InformingScience.org to request redistribution permission.
For example, approximately two-thirds of research outputs in Australia have been attributed to research students (Pearson, Evans, \& Macauley, 2008). Accordingly, maintaining a strong doctoral research culture is crucial for the well-being of universities. In this paper we discuss how locally developed measurement tools (Sampson, Johnston, Comer, \& Brogt, 2016) can be used to effect change with respect to research 
culture and the provision of resources for doctoral candidates. We discuss a number of developments and interventions introduced at the University of Canterbury, driven in response to institutionally developed surveys.

The changing nature of doctoral candidature and candidates necessitates adjustment and flexibility from supervisors, departments, and institutions to provide optimum support. Attitudinal and organizational shifts with regard to expectations and resourcing are required, particularly when moving to support candidates arriving from non-traditional backgrounds, who have varied career aspirations, and who hold differing motivations for undertaking doctoral study. Supervisors have to consider the broader needs of their students, and institutions need to address the breadth of doctoral education and the implications for resource allocation. Changing well-established doctoral degree structures, while retaining high research quality, also requires sensitivity to, and engagement with, individuals and disciplinary structures. This is inherently local and is deeply embedded within the specific context and culture of individual institutions. However, nationally and internationally, the emphasis on evaluating the doctoral experience is in the form of benchmarking surveys and associated institutional league tables. These instruments do have a role in higher education's overall quality assurance framework but lack the resolution and sensitivity to generate data to address specific local needs. In this paper, we demonstrate the value of tailor-made institution-based survey instruments to gather data that can be used to effect meaningful and timely change in the postgraduate research experience.

\section{Changing PhD Experiences}

The number of doctoral candidates is increasing worldwide. For example, in Australia the number of doctoral students in 2010 was 186\% higher than that in 2000; in the United States the number in 2011 was over double that in 2009. Students come from a broader mix of entry pathways, educational, occupational, cultural, and ethnic backgrounds, creating a more diverse doctoral student body. This diversity enriches the university culture but also brings challenges that include greater requirements for flexibility from supervisors and institutions in supporting doctoral candidates (e.g., Bourke, Holbrook, Lovat, \& Farley, 2004).

Accordingly, the needs of current and future doctoral candidates extend beyond disciplinary expertise and skills; the $\mathrm{PhD}$ student now requires and seeks more from the host institution than the opportunity to become an expert in a specific disciplinary domain. Employers are likewise looking for more in doctoral graduates than scholarly expertise in a specific disciplinary area (Jackson \& Michelson, 2015), looking for doctoral graduates with "soft" skills in addition to their disciplinary expertise, skills such as teamwork, project management, and advanced communication skills. Offering expert disciplinary supervision and guidance in a specific research project and thesis writing are necessary but not sufficient for institutions to stay competitive in recruiting and graduating high quality doctoral candidates. The outcome of the doctorate includes both the record of a completed original research presented within a doctoral thesis and the formation of a well-trained, independent researcher (Park, 2007). While the thesis offers the most concrete evidence of project completion, it does not encompass the full doctoral experience; the emphasis is shifting toward greater consideration of the person - the independent researcher - as the primary product of doctoral study (Roberts, 2002).

In addition to disciplinary expertise, the award of the $\mathrm{PhD}$ identifies the individual as having a range of critical thinking and flexible problem solving skills. Success in recruiting, retaining, and graduating doctoral candidates increasingly requires institutions to provide suitable opportunities for fostering transferable skills: "The $\mathrm{PhD}$ has also changed over time so that, irrespective of their degree, research students now experience and expect structured research training as part of their programme" (QAA, 2011). This becomes vital as doctoral graduates increasingly seek professional employment outside academia. Only a minority of doctoral students now enter academic 
careers (Neumann \& Tan, 2011). Consequently, producing versatile graduates with transferable skills will reap long-term reputational and recruitment benefits for an institution (Cargill, 2004; Golovushkina \& Milligan, 2012; Manathunga, Pitt, \& Critchley, 2009).

\section{Current Postgraduate Survey Approaches}

Large scale surveys, such as the Postgraduate Research Experience Questionnaire (PREQ) administered to upward of 6,000 graduates annually from approximately 40 different tertiary education providers throughout Australia (Graduate Careers Australia [GCA], 2011) and The Postgraduate Research Experience Survey (PRES) that gathers feedback from thousands of postgraduates across multiple institutions in the United Kingdom every two years (Hodson \& Buckley, 2011), are used for institutional benchmarking. Benchmarking does, however, assume that the items used to measure practice are consistent enough with the host institution's own experiences to provide meaningful comparisons. Organisational cultures in higher education are, however, as diverse as their stated aims and objectives, enrolments, policies, research foci, and teaching strengths would suggest (Lomas, 1999). Even if key practices contributing to a positive student experience can be identified, outcomes of such practices are largely contingent on the organizational (Lomas, 1999) and socio-cultural (Brogt \& Comer, 2013) settings of each institution. As a result, a benchmarking tool is likely to be too generic and summative to provide actionable results; it simply does not have the resolution and specificity needed to provide data tailored to the varied organisational structures of individual academic institutions. While benchmarking instruments have a role in the overall quality assurance framework of higher education, a tailor-made instrument is better suited to fulfil academic development needs through the provision of highresolution, targeted data that can be used directly to inform changes in operations. A locally designed, administered, and analysed survey offers a responsiveness and malleability not offered by cross-institution surveys for which amendments to items have to be agreed upon by a number of institutions and/or a management board which inevitably leads to slow response times and compromises in the changes made. There is also greater opportunity in locally developed surveys to engage stakeholders in the development and revision of surveys which in turn increases engagement with the measures (Möller, 2006) and embeds it within the academic development culture of the institution; accordingly, the bespoke instrument at the University of Canterbury has been developed to complement benchmarking exercises already undertaken.

In addition, many of the major benchmarking surveys are returned by students at the completion of (or exit from) their qualification. This raises issues connected with reliance on respondents' memories or subsequent re-evaluations of issues at exit, rather than focused reflections concerning their ongoing experiences. Further, collecting data from students only at the end of the qualification means that they do not have 'an active voice' during their candidature. This latter point is vital from an academic development perspective because it limits the impact of any changes made in response to postgraduate feedback to follow-on generations of research students. In contrast, a cross-sectional survey of all enrolled doctoral students allows for timely interventions that can benefit the actual respondents.

The use of a single tool to address both benchmarking and academic development agendas will fall short since it attempts to fulfil two different purposes. We argue that institutions need to employ instruments appropriate to purpose. Accordingly, there is a need for both benchmarking surveys and academic development targeted instruments within the research student domain. We developed a bespoke survey to assess the on-going experiences of current research students (the UCPEQ; Sampson et al., 2016) and complement existing exit and alumni surveys. The UCPEQ serves as a formative tool, based within an academic development agenda, providing detailed information that can, and has, changed supervision practices and the research experience for students, as detailed below. 


\section{The University of Canterbury Postgraduate Experience Survey (UCPEQ)}

The first iteration of the UCPEQ, drew heavily on the work of Graduate Careers Council of Australia (2002) and Ainley (2000) to provide the basis for areas of exploration. Since that time, it has evolved through six iterations between 2004 and 2014 and has been administered to all enrolled doctoral candidates every two to three years, with data collated at the university and the department level. The UCPEQ assesses areas acknowledged as critical for the research candidate experience (McInnis, Griffin, James, \& Coates, 2001), particularly the provision of a supportive environment for research, the importance of the candidate-supervisor relationship, and the ability of students to achieve their goals and aspirations. Questions from the latest UCPEQ iteration related to the research environment and the student-supervisory relationship are included in Appendix $\mathrm{A}^{1}$. Responses represent the on-going experiences of doctoral candidates and provide opportunities to make changes designed to benefit both those same respondents and also future students. All of the responses received are anonymous and treated as confidential. In order to ensure that no students can be identified, all data points presented represent responses collated across a minimum of ten respondents. The number of respondents in each iteration of the survey varied between 500 and 800 students, a response rate exceeding $65 \%$ in each iteration (Sampson et al., 2016). Given the high response rate, the postgraduate research office and the academic development staff have strong evidence of the views of our doctoral candidates and can use these data to drive an effective academic development agenda. Changes in supervisory practices and candidate resourcing occasioned by initiatives developed in response to feedback from these surveys ultimately enhances the research outcomes and reputation of the university itself, as evidenced below.

While the important role of thesis supervisors and the student-supervisor relationship is acknowledged (Barnes \& Austin, 2009; Girves \& Wemmerus, 1988; Grant \& Graham, 1999), with a large number of items being devoted to this topic in the UCPEQ, these responses provide the views from only the student perspective. Accordingly, a parallel survey of thesis supervisors is periodically undertaken to provide information on the provision of practices and resources from the perspective of the supervisor. Comparing the responses from students and supervisors assists in the development of appropriate interventions, especially with respect to identifying whether the desired support is available but poorly communicated. These data have also been used in both development of interventions and in supervisor training.

\section{Application of Survey Feedback}

Having elicited feedback, it is incumbent on those in relevant positions to use the information gathered to develop and enhance the candidate experience. This should involve on-going development and promulgation of effective programmes and the identification and response to shortcomings through appropriate interventions. In developing good practice around thesis supervision, acknowledgement of positive aspects of the postgraduate experience is critical for successful academic development initiatives. Identifying strengths, and ways in which those can be further enhanced, minimizes slippage on the stronger aspects of the research experience while attention is being paid to weaker areas. Just as importantly, good feedback practice includes recognition of positive performance (Bitchener, Basturkmen, East, \& Meyer, 2011; Nicol \& MacfarlaneDick, 2006) and is likely to increase receptiveness to constructive criticism concerning weaker areas by individual supervisors and by their departments and programmes (Hyland \& Hyland, 2001).

\footnotetext{
${ }^{1}$ A full copy of all the UCPEQ questions and response options can be obtained from Kaylene Sampson (kaylene.sampson@,canterbury.ac.nz).
} 
Targeted dissemination of the UCPEQ findings is critical for effecting change. Some issues should be dealt with at the university level, but others need more specific targeting and are best addressed at the most immediate level. For example, department heads have oversight for individual supervisor workloads and resource allocations and, hence, have the ability to effect meaningful changes in response to the recommendations derived from the survey responses.

Providing department-level analyses also provides opportunities for comparison between similar disciplines that can be more meaningful than comparison with global university, or college, level means, which can fail to capture some key disciplinary differences. Such comparisons have indeed led to similar departments working together, sharing experiences and good practice examples. For example, in the 2010 survey two large departments in the same college had almost mirror-image candidate responses in terms of the perceived strengths and weaknesses of the departments. One department was rated as very strong on providing support to the student, encouraging scholarly networking and presentations at conferences, but appeared relatively weak on nonacademic support (including career discussions) and providing effective feedback on work submitted. The other department was very strong on the provision of effective feedback and nonacademic support and career discussions but relatively weak on encouraging networking and scholarly presentations. Discussions between the departments, facilitated by the Dean of Postgraduate Research, resulted in the sharing of good practices and in the subsequent surveys there were commensurate changes with each department showing improvements in their previously weaker items. Here, the UCPEQ findings drove facilitated collaborative exchange between similar departments to enhance the experience of doctoral candidates in each.

Table 1. Items that most strongly predict overall student satisfaction with the postgraduate experience; $r>.54, p<.001$ (UCPEQ, 2014).

\section{Item:}

I have had productive conversations with my supervisors about my educational goals

I am satisfied with my student-supervisor agreement

I have had productive conversations with my supervisors about my career goals

I have a clear understanding of the schedule for progress and deadlines associated with my research

Usefulness of feedback from my supervisors regarding my research process

Supervisors' ability to communicate knowledge in order to assist my research

Levels of communication between members of my supervisory team

My experience with my primary supervisor

As the vast majority of public universities have limited resources available, it is crucial to clearly identify key areas for development or intervention, at both the university and department levels. Attention is focused on maximizing engagement with areas that have been shown in past research and in UCPEQ iterations (McInnis et al., 2001; Sampson et al., 2016) to best predict overall student satisfaction with the postgraduate experience. We regressed scores on the individual survey items onto the overall satisfaction rating. The items that most strongly predicted overall student satisfaction are shown in Table 1. These items were then the focus in designing interventions to improve the student-supervisory experience, as detailed below. In addition, factors with lower satisfaction scores across the entire university are identified as requiring a university-wide intervention; often these are around global resourcing issues (e.g., conference funding allowances). At the department level an overall picture of performance is created by looking at the percentage of 
items on which there is strong agreement (positive responses) or disagreement (negative responses). This analysis extends to the percentage of items on which performance is higher or lower than the university mean, as well as the shifts in percentages for items showing marked improvement and deterioration since the last survey iteration. The format of the collation of responses and feedback is shown in Appendix B.

\section{Reflection on Feedback Initiatives: Providing Evidence for Action}

Responses from the UCPEQ call for action to be taken at both university and department levels to improve the doctoral student experience. These actions can be categorized into two broad but non-exclusive types: (a) provision of resources to support identified student needs, and (b) changes to behaviour/culture around doctoral candidature. We discuss examples of each at individual supervisor, department and university levels.

\section{Provision of resources}

Once awareness was raised from responses to the UCPEQ, a number of resourcing issues could be easily addressed. For example, the university increased standard data storage capacity limits for all doctoral students and provided an email address identifying the status of the students as postgraduate research students. These simple and relatively inexpensive changes were made within months of data collation and had a large impact on students, with positive feedback to the change in email addresses reported by the postgraduate student association as demonstrating the university's recognition of their status and distinction from undergraduates' email addresses. In such cases the survey simply provides a forum for which the collective voice of students can be heard, which is more likely to result in action than were the same issue(s) raised individually by students across campus.

Other changes, such as creating an environment of greater inclusiveness of students within the research culture of departments, are more complex, resource demanding, or time consuming to implement, maintain, and continue to develop.

\section{Thesis Supervision Training}

The student-supervisor relationship is a critical component of success and satisfaction with the doctoral experience (Barnes \& Austin, 2009; Girves \& Wemmerus, 1988; Grant \& Graham, 1999; Park, 2007; Zhao, Golde, \& McCormick, 2007). Provision of support to research students must be considered in conjunction with that given to supervisors. Providing support and guidance to doctoral candidates requires that supervisors have the skills, resources, and time in order to do so. Early iterations of the UCPEQ identified concerns amongst candidates with regard to core supervisory practices, including the student-supervisor agreement and clarity of expectations within the relationship. This latter is of particular relevance given the importance of congruity in student and supervisors' perceptions of supervision and for the quality of the supervisory relationship and research outcomes (Malfroy \& Webb, 2000). Importantly, many of these concerns were ones that are strong predictors of overall student satisfaction in our survey, as shown in Table 1 .

A similar issue emerged through the 2007 survey of supervisors, in which only $45 \%$ agreed that there was sufficient support for new supervisors, and 50\% agreed that there was adequate support for all supervisors. Accordingly, there has been a complete overhaul of support offered to supervisors. The existing, one-off 3-hour new supervisors' orientation session was replaced in 2012 by a mandatory 3-4 month series of workshops providing academic development sessions on supervision for all academics new to the university and an ongoing series of workshops open to all su- 
pervisors. The impact of these workshops has now been recognized by their development into a full academic course as part of a postgraduate certificate in tertiary teaching.

Relevant factors identified from responses to the UCPEQ and the supervisors' survey were key drivers in the design and development of specific modules in the new supervisor training programme. This curriculum now includes the following: recruiting good doctoral candidates; establishing and clarifying candidate and supervisor expectations; the role, and modes, of supervision; giving effective feedback; and assisting students in career planning and skill portfolio development. Assessing and evaluating the factors associated with effective supervisory practice requires addressing the behaviours and attitudes of both candidates and supervisors (Acker, Hill, \& Black, 1994); accordingly, complementary workshops have been provided for students.

It will take some time for the impact of new thesis supervisor training changes to be fully reflected in the UCPEQ results, but the 2014 iteration of the survey has already shown gains on some of these key issues (see Figures 1 and 2). Particularly noteworthy are the steep increases in satisfaction since 2012 with items relating to feedback, which was a new module introduced to supervisor training in 2012, and relating to communication with supervisors, which is a key feature in the training programme. Additionally, the most recent supervisor survey saw an increase to $60 \%$ of respondents who were in agreement that there is adequate support for new supervisors and provision of ongoing support for supervisors.

The UCPEQ allowed for a detailed understanding of the students' needs, and through development of appropriate and targeted workshops and training modules for students and supervisors we were able, within two years, to demonstrate a positive impact for both students and supervisors. Feedback from students in regard to supervisory practice enabled the provision of training and support to supervisors, the effectiveness of which was demonstrated by increased satisfaction by students with regard to the same issues.

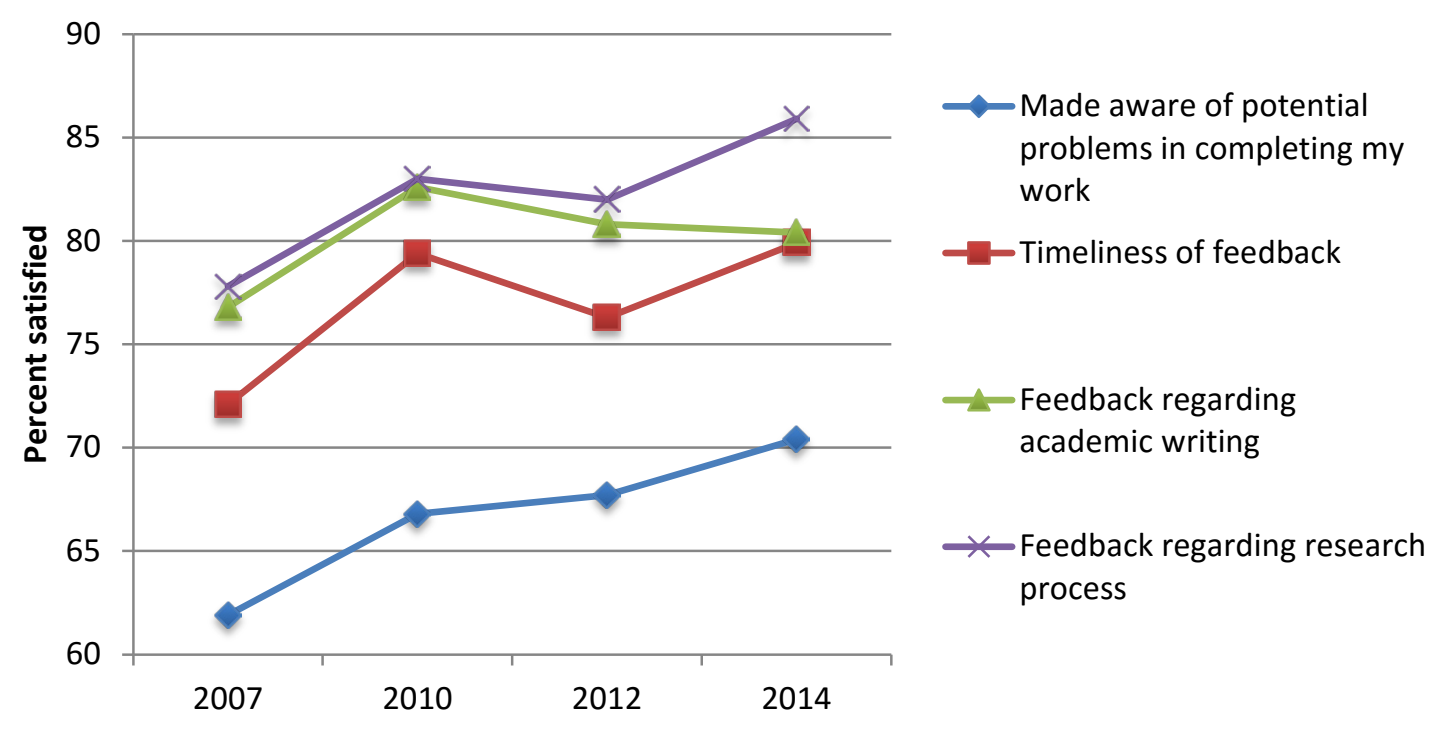

Figure 1. Student satisfaction levels on feedback-related items from the UCPEQ 


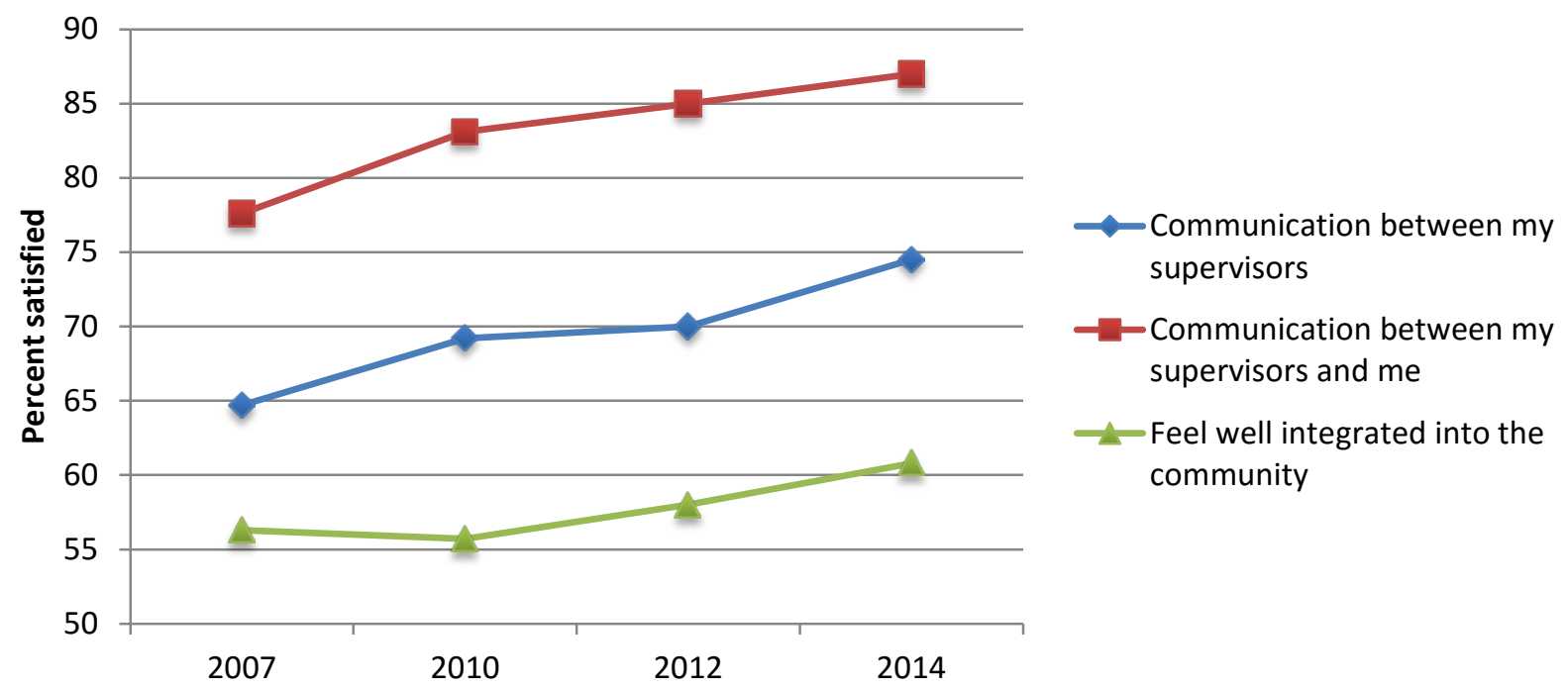

Figure 2: Student satisfaction levels on communication- and connection-related items from the UCPEQ

\section{Transferable skills}

Candidates' feedback from the UCPEQ reflects needs, noted worldwide, regarding the inclusion of transferable, generic skills in doctoral pathways. The changing nature of the $\mathrm{PhD}$ requires inclusion of such skills training and development components. UCPEQ findings demonstrate that students consider it important to have opportunities for extending their skills in a variety of general domains, such as in the communication of research, through scholarly and professional networking, by gaining research skills (especially statistical ones), time and project management, and via career planning. Increasing recognition of the importance of providing opportunities for candidates to acquire transferable skills beyond their discipline has led to the provision, within many doctoral programmes, of skills training and professional development initiatives (Cargill, 2004; Golovushkina \& Milligan, 2012; Manathunga et al., 2009). Some universities now use these developments to market their PhD programmes, promoting their offerings as superior and therefore establishing a competitive advantage for their doctoral graduates.

The University of Canterbury developed, and continues to evolve, a range of skills training sessions for doctoral candidates, many of which have been developed in response to feedback from the UCPEQ as detailed above. Sessions also provide invaluable opportunities to engage with others on the doctoral journey and exchange ideas, tips and provide mutual support (Hamerton \& Fraser, 2014). They also enhance networking and connection of candidates and academics across campus, thereby creating a university doctoral community and research culture and promoting the development of inter-disciplinary networks and research (Deem \& Brehony, 2000; Pilbeam, Lloyd-Jones, \& Denyer, 2013).

Our skills sessions supplement rather than replace the disciplinary expertise of the thesis supervisors and are aimed at reducing workload for supervisors whilst enhancing the learning experience for students. For example, while many supervisors are disciplinary experts and author multiple research papers, they frequently are not skilled at teaching students about writing (Paré, 2011), and they may not be attuned to the discursive and structural elements of writing within their discipline (Trivett, Skillen, \& James, 2001). Writing skills advisors can provide this expertise, regardless of field of study. Consequently, modules have recently been included in both the new thesis supervisors' course and in the doctoral student "Surviving your Thesis" seminar series on aspects of writing (e.g., research proposals; thesis chapters; journal articles); for more details of 
one writing workshop see Comer, Clement, Brogt, and Obel (2013). Similarly, supervisors may need support in teaching advanced research methods, data analysis, and statistics to doctoral candidates - the UCPEQ data over several iterations has revealed these areas identified by students as relatively low in support. Accordingly, a series of workshops and seminars around this topic was introduced in 2013 for doctoral students. A challenge in further developing these skills sessions is to work with supervisors to ensure that they recognise the benefits for their students from increased training opportunities (Glibert, Balatti, Turner, \& Whitehouse, 2004). Candidates' participation and engagement with skills' training is heightened when their supervisors actively encourage rather than (implicitly or explicitly) discourage involvement (Carter \& Laurs, 2014).

\section{Career Planning}

With completion of a $\mathrm{PhD}$ previously seen almost solely as a pathway to an academic career, provision of career advice was seen as a component of supervision, introducing students to key components within an academic career (e.g., research outputs, teaching, grant application writing). With a more diverse doctoral cohort pursuing a far wider range of career goals, the ability of supervisors to provide adequate career guidance and networking opportunities has declined. Repeated iterations of the UCPEQ and the university exit survey have indicated low satisfaction with the provision of career guidance and support, consistent with international literature (Carter \& Laurs, 2014). It is vital that provision of good career advice is made available to doctoral candidates; poor career outcomes for doctoral graduates substantially raises the risk of negative impacts on the institution's reputation for doctoral education.

Consequently, and in response to candidate feedback, we have introduced two workshops for doctoral candidates, each offered twice per year. The first - Career Planning - is targeted for students early in their candidature to ensure that they begin thinking about career options and hence opportunities (e.g., networking) that should be pursued through their candidature. The second workshop - Job Market Readiness - is more directly focused on presenting one's skills and attributes to potential employers. Feedback on the exit survey indicates an improvement in agreement by students with regard for the provision of support for career development ( $27 \%$ in 2013 ; $39 \%$ in 2014), but this remains an area requiring more intervention. Future developments include the introduction of a "needs analysis" approach for doctoral students at the beginning of their studies to determine the opportunities and experiences they would benefit from during their candidature.

In another initiative addressing this concern, we recently introduced a searchable database in which doctoral candidates can locate profiles of doctoral graduates by discipline, by supervisor, or by career. All the profiles are supplied by University of Canterbury doctoral graduates: they describe their current jobs, discuss how these are linked to their doctoral studies, comment on aspects of their doctorates they use most, and consider what they might have done differently were they to start the doctorate over again. In addition, some graduates have provided contact details so that current candidates may connect with them for further information or introductions. These initiatives further increase professional networking opportunities for doctoral candidates, which is another area identified in the UCPEQ as requiring development.

\section{Creating a supportive research environment for doctoral candidates}

The research environment is complex and includes candidates' engagement with their departmental community (e.g., through internal research seminar series and presentations), the wider research culture of the discipline (e.g., through presentations and collaborations both inside and outside the university), and scholarly and professional networking. While additional resources might help aspects of these concerns (e.g., funding for conference attendance), long-term solutions involve greater changes by individual supervisors and in department and university practic- 
es. In repeated iterations of the UCPEQ, continued relatively low levels of agreement regarding perceived integration into departmental research cultures both demonstrates the challenges associated with attitudinal change and highlights its necessity.

Looking at the university mean ratings for departmental integration conceals a number of successful interventions at departmental level. In the latest iteration of the UCPEQ at least one department in each of the five colleges of the university showed an improvement of $20 \%$ or higher in student agreement with statements that they are integrated into their department since the previous UCPEQ iteration only two years previously. This demonstrates that effective interventions can be made in this area with rapid positive impact on the student experience. Within the same period, however, a number of departments have also shown similar sized declines in the percentage of student agreement with these items; hence the relative stability of the overall mean. Efforts must focus on maintaining the improvements made in some departments and sharing of good practice with those departments not yet showing improvement.

Attitudes are relatively stable, and slow and resistant to change (Prislin, 1996). A slow pace of change in attitudes toward supervision can result in unsatisfied postgraduates and challenges efforts to improve and maintain the institution's reputation. In attempting to affect shifts in attitude and enact positive changes for current students, our approach has focused on behavioural change - positive change in relevant behaviours can eventually lead to (indirect) attitude change (Higgins \& McCann, 1984; McCann \& Hancock, 1983), which in turn influence future behaviour (Fazio, 1986). Relatively small behavioural changes can have a very positive impact on student satisfaction.

The strategies detailed below are examples of those that have been employed by different programmes to increase integration of doctoral candidates into their department research cultures and thereby enhance its vibrancy. Different approaches have been taken by different departments. Consequently, the impact of each individual behaviour is difficult to quantify, but the commonality across them is the aim of enhancing interactions of doctoral students with both academic staff members as well as other postgraduates.

Marked shifts in perceptions of the research environment can be brought about through relatively minor changes in on-going practices. For example, in 2010 less than a third of doctoral candidates in one school indicated feeling integrated into their departmental community. The department singled this out as an issue to be addressed, considering it especially important in the context of a relatively small programme located in a somewhat isolated locale. Working with staff from the academic development unit, the department introduced three on-going activities: a departmental barbeque each term, a monthly feedback forum between postgraduates and staff (academic and general), and students were taken for coffee to the department break room by their supervisors. In the next UCPEQ iteration nearly two-thirds of students reported feeling integrated. Relatively small behaviours can, then, have a noticeable impact on student perceptions of integration.

\section{Laboratory/Research Group Meetings}

Disciplinary differences in supervisory practices, and differences in the intersection between student and supervisor research, and in research methodologies and equipment use can result in disparities in the sense of togetherness of groups of students and supervisors. Where students and supervisors spend extended time together, for example working with shared resources in a laboratory environment, it is easy to create a team environment and to share research ideas and challenges. Where individually oriented or library- or archive-based research is more common (e.g., in humanities) this sense of integration is harder to achieve, and students are in danger of feeling isolated and having fewer opportunities for networking. These disciplinary differences are apparent in responses to many such issues in the UCPEQ. 
Previous research has explored how research teams, traditionally more typical of supervisory practices in the sciences and engineering fields, often foster enhanced networks for postgraduates (Sampson \& Comer, 2011). Such networks can also, however, be fostered in other domains. In response to comments from an academic that "lab groups" do not exist outside of science disciplines, the Dean of Postgraduate Research suggested she create her own research group disciplines ("just put a sign saying 'lab group' on a meeting room door"), bringing together her students to share their ideas, issues and successes. Even though they were conducting diverse research projects, the Dean argued that this sharing could help address potential problems of isolation, provide peer support, and expose students to varied viewpoints, perspectives and methods. The following year, this academic offered a presentation at the University's Teaching Week describing the positive impacts of her lab group innovation, describing her students were more integrated, were sharing ideas, and were providing each other with peer support in a newly vibrant research community. Subsequently, her entire department has introduced a similar research group approach. We expect such developments - which involve limited resources and time - will be reflected in more positive evaluations regarding integration in the next UCPEQ iteration.

\section{Departmental Seminars}

One of the easiest ways to be noticed and become integrated within a department is through attendance at, and participation in, departmental colloquia. Increasing student engagement with department seminars is one key way to address student concerns, expressed in the UCPEQ, regarding inadequate departmental integration. Attending seminars brings students into the department community and increases possibilities for networking, both within the department and with external speakers. UCPEQ responses indicate, however, that students need to be specifically invited, accompanied, and welcomed to seminars, not just sent a generic email with the schedule. Interestingly, this is an item on which there is discrepancy between the student and supervisors perceptions. Supervisors considered a generic email notice for seminars to be a specific invitation to students to attend. After discussion and putting themselves in the place of the students, however, supervisors agreed that, especially for new students, this was not sufficient and that the situation could be intimidating. It is hoped that this enhanced awareness will improve student experience which will reflect positively in the next survey iteration.

\section{Presentation of Research}

UCPEQ data have consistently shown that doctoral candidates experience difficulty in obtaining funding to attend conferences, especially those involving international travel. In view of the low prospects for increased conference funding in the immediate future, postgraduates and supervisors need to find alternative ways to create opportunities for practicing important, and transferable, communication and networking skills. Many departments now run annual conference days for thesis students to present their research, a number of which are associated with external sponsors. These initiatives are to be commended, and other departments have been encouraged to introduce similar events. Concerns about a lack of being integrated into the department's research environment highlighted in UCPEQ data could be further reduced by broadening the scope of such events to combine presentations by postgraduates and academics, such that staff and student research is integrated in the department's research culture and undertakings; some departments are doing this in 2015.

Partially in response to UCPEQ feedback, the Dean of Postgraduate Research facilitates a number of university-wide fora for students to showcase their research. These provide opportunities for postgraduates to present their work to wider, less disciplinary-specific audiences (a skill required in almost every employment situation), including to non-university individuals or groups (e.g., local industry or government representatives). While not the equivalent of national or international conferences, they provide opportunities to present and receive feedback in a supportive envi- 
ronment and to network with professionals outside their usual settings. National and local media (television, radio, newsprint) have reported on student research following these university presentations. As new PhDs increasingly move into non-academic professional employment, these venues connect participants with the broader audiences they need to anticipate addressing. Additionally, such local events can offer candidates with opportunities to become involved in organizing conferences, for example reviewing abstracts, arranging event programmes, and inviting and introducing keynote speakers - all helpful to the development of transferable skills. Increased use of social media at international conferences can also enable some engagement by doctoral students "at a distance" and should be encouraged.

\section{Conclusions}

The need to monitor and evaluate the experience and needs of doctoral students and supervisors, and to respond to the information received, is incumbent on institutions. Failure to do so presents a risk to a university's research reputation and future recruitment of postgraduate students. While it is unlikely that all student expectations and desires can be met, giving students a voice to express their opinions, both positive and critical, and demonstrating a considered and timely response is important in enhancing student satisfaction. This, then, requires the development and employment of suitable survey measures and an engaged response to the data gathered. For this to be a worthwhile endeavour, however, there needs to be strong engagement with the process by all stakeholders - students, supervisors, Heads of School and university management. We have argued that data-driven and locally-based approaches, coupling institutional research with academic development, are required for generating buy-in from academic supervisors and the institution to initiate and sustain change. Locally developed scales and local analysis and responses make the survey, and its findings, more meaningful and tangible to stakeholder.

No matter how clearly information and analyses from survey instruments such as the UCPEQ are presented and communicated within a university, closing the feedback loop requires more than leaving academics to fend for themselves. Involvement of academic developers, heads of department, and deans of postgraduate research are essential to effect change.

Many changes that can markedly improve the postgraduate experience are not costly or resource intensive. Once issues are highlighted, relatively minor shifts in supervisory practices and department- and university-level actions can result in direct and measurable improvements in the student experience and overall research environment. While the examples included in this paper are specific to the University of Canterbury, the general methods and interventions are readily applicable in other institutions. Indeed many of the concerns that doctoral students have around their research experience and supervision are shared across institutions and countries (Apsland, Edwards, O’Leary, \& Ryan, 1999; Moses, 1984; Spear, 2000). As emphasized earlier, however, it is important that institutions consider their specific context and students and tailor both surveys and interventions accordingly. Greater engagement by students, supervisors and management will be achieved when the survey, and its outcomes, are perceived as personally relevant.

Long-term and on-going progress in initiatives arising from locally designed bespoke instruments such as the UCPEQ, in combination with exit and alumni surveys, requires the sustained and coordinated support of a range of university offices. Implementing and maintaining progress in these areas involves (but is not limited to) direct support for supervisors, postgraduate research offices and their leaders, academic development units, research librarians, IT staff, and academic/learning skills units. Working together, virtuous circles can be achieved to enhance the doctoral student experience and accordingly enhance the reputation of the institution. 


\section{References}

Acker, S., Hill, T., \& Black, E. (1994). Thesis supervision in the social sciences: Managed or negotiated? Higher Education, 28, 483-498.

Ainley, J. (2000). The 1999 postgraduate research experience questionnaire. Evaluations and Investigations Programme, Higher Education Division, Department of Education, Training and Youth Affairs, Canberra.

Apsland, T., Edwards, H., O'Leary, J., \& Ryan, Y. (1999). Tracking new directions in the evaluation of Postgraduate Supervision. Innovative Higher Education, 24, 127-147.

Barnes, B. J., \& Austin, A. E. (2009). The role of doctoral advisors: A look at advising from the advisor's perspective. Innovative Higher Education, 33, 297-315.

Bitchener, J., Basturkmen, H., East, M., \& Meyer, H. (2011). Best practice in supervisor feedback to thesis students. Research report, Ako Aotearoa, Wellington, New Zealand.

Bourke, S., Holbrook, A., Lovat, T., \& Farley, P. (2004, November). Attrition, completion and completion times of $\mathrm{PhD}$ candidates. In AARE annual conference, Melbourne (Vol. 28).

Brogt, E., \& Comer, K. (2013). Interpreting differences between US and NZ university students' engagement scores as measured by the NSSE and AUSSE. Assessment and Evaluation in Higher Education, 38(6), 713-736.

Cargill, M. (2004). Transferable skills within research degrees: A collaborative genre-based approach to developing publication skills and its implications for research education. Teaching in Higher Education, 9, 83-98.

Carter, S., \& Laurs, D. (2014). Developing generic support for doctoral students: Practice and pedagogy. Routledge: London.

Comer, K., Clement, J., Brogt, E., \& Obel, C. (2013) Postgraduate writing for publication workshops: Preparation for the past or for the future? TEXT: Journal of Writing and Writing Courses Special Issue 21 (Scores from another ground: Writing in New Zealand).

Deem, R., \& Brehony, K. J. (2000). Doctoral students access to research cultures - Are some more equal than others? Studies in Higher Education, 25, 149-165.

Fazio, R. H. (1986). How do attitudes guide behavior. Handbook of motivation and cognition: Foundations of Social Behavior, 1, 204-243.

Gilbert, R., Balatti, J., Turner, P., \& Whitehouse, H. (2004). The generic skill debate in research higher degrees. Higher Education Research and Development, 23, 275-288.

Girves, J. E., \& Wemmerus, V. (1988). Developing models of graduate student degree progress. The Journal of Higher Education, 163-189.

Golovushkina, E., \& Milligan, C. (2012). Developing early stage researchers: Employability perceptions of social science doctoral candidates. International Journal for Researcher Development, 3, 64-78.

Graduate Careers Council of Australia. (2002). Postgraduate research experience questionnaire 2000. Parkville, Victoria: Graduate Careers Council of Australia.

Graduate Careers Australia (GCA). (2011). The postgraduate research experience 2010. A report on the postgraduate research experience questionnaire. Retrieved September 13, 2013, from http://www.graduatecareers.com.au/wp-content/uploads/2012/01/gca002728.pdf

Grant, B., \& Graham, A. (1999). Naming the game: Reconstructing graduate supervision. Teaching in Higher Education, 4, 77-89.

Hamerton, H., \& Fraser, C. (2014). Writers' retreats: An opportunity for improving academic writing and publication success. EDULEARN14 Proceedings, 4482-4488. 
Higgins, E. T., \& McCann, C. D. (1984). Social encoding and subsequent attitudes, impressions, and memory: "Context-driven" and motivational aspects of processing. Journal of Personality and Social Psychology, 47, 26-39.

Hodson, L. \& Buckley, A. (2011). Postgraduate research experience survey. Retrieved June 3, 2013, from http://www.heacademy.ac.uk/assets/documents/postgraduate/PRES_report_2011.pdf

Hyland, F., \& Hyland, K. (2001). Sugaring the pill: Praise and criticism in writing feedback. Journal of Second Language Writing, 10, 185-212.

Jackson, D., \& Michelson, G. (2015). Factors influencing the employment of Australian PhD graduates. Studies in Higher Education, 40(9), 1660-1678.

Lomas, L. (1999). The culture and quality of higher education institutions: Examining the links. Quality Assurance in Education, 7, 30-34.

Malfroy, J., \& Webb, C. (2000). Congruent and incongruent views of postgraduate supervision. Quality in Postgraduate Research: Making Ends Meet, 165-177.

Manathunga, C., Pitt, R., \& Critchley, C. (2009). Graduate attribute development and employment outcomes: Tracking PhD graduates. Assessment \& Evaluation in Higher Education, 34, 91-103.

McCann, C. D., \& Hancock, R. D. (1983). Self-monitoring in communicative interactions: Social cognitive consequences of goal-directed message modification. Journal of Experimental Social Psychology, 19, 109-121.

McInnis, C., Griffin, P., James, R., \& Coates, H. (2001). Development of the course experience questionnaire (CEQ). Canberra: Department of Education, Training and Youth Affairs.

Möller, O. (2006). Student satisfaction survey: The Utrecht university approach. Tertiary Education Management, 12(4), 323-328.

Moses, I. (1984). Supervision of higher degree students - Problem areas and possible solutions. Higher Education Research \& Development, 3, 153-165.

Neumann, R., \& Tan, K. K. (2011). From PhD to initial employment: The doctorate in a knowledge economy. Studies in Higher Education, 36, 601-614.

Nicol, D. J., \& Macfarlane-Dick, D. (2006). Formative assessment and self-regulated learning: A model and seven principles of good feedback practice. Studies in Higher Education, 31, 199-218.

Paré, A. (2011). Speaking of writing: Supervisory feedback and the dissertation. Doctoral education: Research-based strategies for doctoral students, supervisors and administrators. Springer Netherlands, pp. 59-74.

Park, C. (2007). Redefining the doctorate: A discussion paper. York: Higher education Academy.

Pearson, M., Evans, T., \& Macauley, P. (2008). Growth and diversity in doctoral education: Assessing the Australian experience. Higher Education, 55, 357-372.

Pilbeam, C., Lloyd-Jones, G., \& Denyer, D. (2013). Leveraging value in doctoral student networks through social capital. Studies in Higher Education, 38, 1472-1489.

Prislin, R. (1996). Attitude stability and attitude strength: One is enough to make it stable. European Journal of Social Psychology, 26, 447-477.

QAA (The Quality Assurance Agency for Higher Education). (2011). Doctoral degree characteristics. Gloucester: The Quality Assurance Agency for Higher Education, UK.

Roberts, R. (2002). SET for Success. The supply of people with science, technology, engineering and mathematics skills. Retrieved 1/10/12 from http://www.hm-treasury.gov.uk/d/robertsreview introch1.pdf

Sampson, K., \& Comer, K. (2011). Engineering research teams: The role of social networks in the formation of research skills for postgraduate students. International Journal for the Scholarship of Teaching and Learning, 5(1), 14. 
Sampson, K., Johnston, L., Comer, K., \& Brogt, E. (2016). Developing evidence for action on the postgraduate experience: An effective local instrument to move beyond benchmarking. Higher Education Research and Development, 35(2), 337-341.

Spear, R.H. (2000). Supervision of research students: Responding to student expectations. ANU Research Publications. Retrieved from http://hdl.handle.net/1885/41534; http://digitalcollections.anu.edu.au/handle/1885/41534

Trivett, N., Skillen, J., \& James, B. (2001). New partnerships in supporting postgraduate research. In Proceedings of 24th International HERDSA Conference.

Zhao, C. M., Golde, C. M., \& McCormick, A. C. (2007). More than a signature: How advisor choice and advisor behaviour affect doctoral student satisfaction. Journal of Further and Higher Education, 31, 263-281 


\section{Appendices}

\section{Appendix A: UCPEQ - Questionnaire Extract}

We are keen to find out about skills you are developing Please rate the following statements on the scale provided.

(scale: strongly disagree to strongly agree + does not apply).

I have received sufficient training in research methodology to adequately carry out my proposed research

My research experience is further developing my problem solving skills

The process I am undergoing to design my research is effective

My research experience is helping to develop my written communication skills

My postgraduate experience is improving my oral communication skills

I received adequate advice and support for preparing my research proposal

My research experience is improving my ability to think critically about my work

My research experience is improving my ability to organise my own work independently

We would now like to find out about your access to resources. Please rate the following statements on the scale provided.

(scale: strongly disagree to strongly agree + does not apply).

I have sufficient access to computing facilities

The library facilities and services are sufficient to carry out my research

There is sufficient access to financial support for my research activ-

ity

I am able to get appropriate levels of support from technical and/or administrative staff when needed

I have access to a suitable workspace to conduct my research and write it up

I have sufficient access to electronic data storage space for my research needs

I have access to financial resources to attend academic conferences/exhibits

We would like to know about the research culture you are operating in.

Please rate the following statements on the scale provided.

(scale: strongly disagree to strongly agree + does not apply).

I feel well integrated into the department's/school's community

I have been encouraged to publish

I have been encouraged to exhibit, perform or show my artistic or creative works

I have been encouraged to attend staff and/or departmental research seminars

My school/department encourages me to present my work at seminars/ workshops

WITHIN the University

My school/department encourages me to present my work at conferences/ workshops

OUTSIDE the University 
I have opportunities to discuss my research with members of my department outside of my supervisory team and research peers (these can include other students and staff)

We want to find out about your goals and expectations. Please rate the following statements on the scale provided.

(scale: strongly disagree to strongly agree + does not apply).

I am clear about what is expected of the student-supervisor relationship

I am satisfied with my student-supervisor agreement

I have a clear understanding of the schedule for progress and deadlines associated with my research

I have had productive conversations with my supervisors about my educational goals

I have had productive conversations with my supervisors about my career goals

Overall, I am satisfied with the quality of my postgraduate experience

In this section, we want to find out about your experiences with supervision. Please rate the following statements. Please mark "does not apply" in the case where the aspect raised is not applicable to your particular circumstance, e. g. you may not be able to comment on guidance in academic writing if you have not yet commenced writing. Please choose "this does not concern me" ONLY in the case where you are unable to rate this item on the satisfaction scale because it is of such little importance to your experience.

(scale: very dissatisfied to very satisfied + does not apply to me + does not concern me).

Expertise of my supervisory team in my area of study

My supervisors' knowledge of the current literature to support my research

Supervisors' enthusiasm for my research

Support for networking through introductions and professional contacts

Supervisors' ability to communicate knowledge in order to assist my research

The availability of my supervisory team

Usefulness of feedback from my supervisors regarding my research process

Usefulness of feedback from my supervisory team regarding my academic writing

Timeliness of feedback from my supervisory team

Extent to which I have been made aware of potential problems in completing my work

Level of communication between myself and my supervisors

Level of communication between members of my supervisory team

Level of personal (non-academic) support from my supervisory team

My experience with my primary supervisor

My experiences with my secondary supervisor 


\section{Appendix B}

Format 1: Percentage of respondents who agree or strongly agree with each item as a function of college and comparison between the current and previous iteration of the survey. The same reporting is also provided at a departmental level within each college.

\begin{tabular}{|c|c|c|c|c|c|c|c|c|}
\hline \multirow{2}{*}{ Item } & Arts & Bus \& Law & Educ. & Engin. & Sci. & $\mathbf{2 0 1 4}$ & 2012 & $\begin{array}{c}+f \text { change } \rightarrow \rightarrow \\
\%\end{array}$ \\
\hline
\end{tabular}

Format 2: The highest and lowest percentage agreement items for each department are reported and compared with percentages from the relevant college and university.

\section{Areas of greatest strength}

\begin{tabular}{|c|l|l|l|}
\hline General Experience & \multicolumn{1}{c|}{$\begin{array}{c}\text { Department } \\
2014\end{array}$} & $\begin{array}{c}\text { College } \\
2014\end{array}$ & \multicolumn{1}{c|}{$\begin{array}{c}\text { University } \\
2014\end{array}$} \\
\hline 3 items & $\%$ & $\%$ & $\%$ \\
\hline Supervision & & & $\%$
\end{tabular}

Areas of greatest weakness

\begin{tabular}{|c|l|l|l|}
\hline General Experience & \multicolumn{1}{c|}{$\begin{array}{c}\text { Department } \\
2014\end{array}$} & $\begin{array}{c}\text { College } \\
2014\end{array}$ & \multicolumn{1}{c|}{$\begin{array}{c}\text { University } \\
2014\end{array}$} \\
\hline 3 items & $\%$ & $\%$ & $\%$ \\
\hline Supervision & & & $\%$ \\
\hline 3 items & $\%$ & $\%$ &
\end{tabular}

Format 3: For each department and college the number of items for which over $80 \%$ of respondents agreed/disagreed and under $40 \%$ agreed/disagreed were identified, along with the number of items that had increased since the previous survey iteration and that number that were higher than the university mean.

\begin{tabular}{|l|l|l|l|l|l|l|l|l|}
\hline & \multicolumn{3}{|c|}{ Supervisory Items (N=15) } & \multicolumn{3}{c|}{ General Experience Items (N=27) } \\
\hline item & $\begin{array}{l}\#>80 \% \\
\text { agreement }\end{array}$ & $\begin{array}{l}\#<40 \% \\
\text { agreement }\end{array}$ & $\begin{array}{l}\text { \# increased } \\
\text { since 2012 }\end{array}$ & $\begin{array}{l}\text { \# higher } \\
\text { than Uni } \\
\text { mean }\end{array}$ & $\begin{array}{l}\#>80 \% \\
\text { agreement }\end{array}$ & $\begin{array}{l}\#<40 \% \\
\text { agreement }\end{array}$ & $\begin{array}{l}\# \text { increased } \\
\text { since 2012 }\end{array}$ & $\begin{array}{l}\# \text { higher } \\
\text { than Uni } \\
\text { mean }\end{array}$ \\
\hline
\end{tabular}




\section{Biographies}

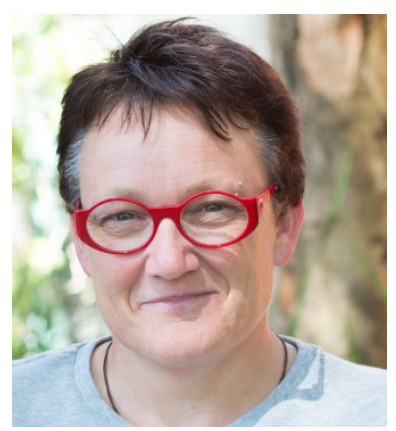

Lucy Johnston is the Dean of Graduate Studies at the University of Newcastle, Australia and formally Professor of Psychology and Dean of Postgraduate Research at the University of Canterbury, New Zealand. Her research interests include student and supervisor experiences in thesis research.

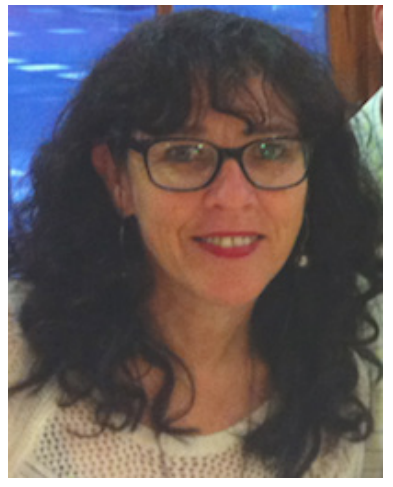

Kaylene Sampson is a Senior Institutional Researcher in the Academic Services Group at the University of Canterbury, Christchurch, New Zealand. Her research interests include the postgraduate student experience and graduate outcomes.

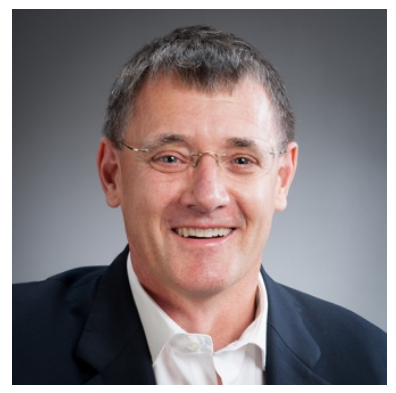

Keith Comer is a Senior Lecturer in writing and literature studies in the School of English and Media Studies at Massey University. He has previously worked in Sweden and the USA in related fields, as well as in academic development in New Zealand.

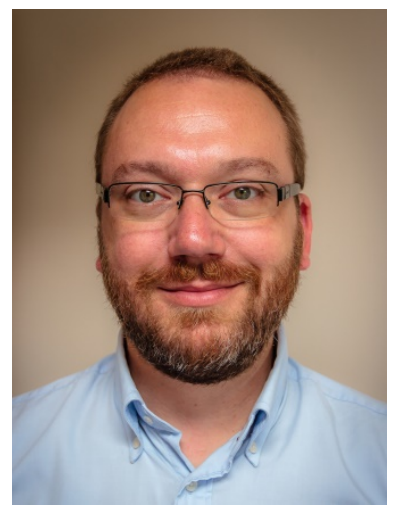

Erik Brogt is a Senior Lecturer in Academic Development at the University of Canterbury. His research interests are in the educational psychology of teaching and learning in university settings and in science education, in particular physics, astronomy and natural hazards. 